International Journal of Wireless \& Mobile Networks (IJWMN) Vol. 6, No. 2, April 2014

\title{
Impact of Client Antenna's Rotation Angle AND HeIght OF 5G Wi-Fi ACCESS POINT ON INDOOR AMOUNT OF FADING
}

\author{
Jehad Hamamra, Hassan El-Sallabi and Khalid Qaraqe \\ Electrical Engineering Department \\ Texas A\&M University at Qatar
}

\begin{abstract}
This paper investigates the impact of antenna rotation's angle at the receiver side and antenna height at transmitter side on radio channel's amount of fading. Amount of fading is considered as a measure of severity of fading conditions in radio channels. It indicates how severe the fading level relative to Rayleigh fading channel. The results give an input to optimize height of $5 \mathrm{G}$ Wi-Fi access point for better link performance for different antenna's rotation angles at receiver side. The investigation covers three different indoor environments with different multipath dispersion levels in delay and direction domains; lecture hall, corridor, and banquet hall.
\end{abstract}

\section{KEYWORDS \\ Reconfiguring Antenna, Radio Channel, Amount of Fading, Channel Model}

\section{INTRODUCTION}

The coming generations of Wi-Fi wireless networks 5G-Wi-Fi, IEEE 802.11ac [1] and WiGig (Millimeter Wave), IEEE 802.11ad are expected to provide extremely high data rate communication services. These technologies are mainly required to support advanced services such as efficient real-time video communication for users. Vast majority of mobile users usually spend most of their time in indoor environments. In fact, these indoor environments are miscellaneous and diverse. Communication system performance depends on radio channel characteristics of each indoor sceanario. The radio channel characteristics are function of distribution of scatterers, mobility in the channel at both ends of the connection link, bandwidth, frequency, and antenna's position of both transmitter and receiver and their radiation patterns as well.

The IEEE $802.11 \mathrm{ac}$ has a mandatory operation at the $5 \mathrm{GHz}$ frequency range and bandwidth of 80 $\mathrm{MHz}$ with $160 \mathrm{MHz}$ available optionally. The interference level near $5 \mathrm{GHz}$ is less than that at around $2 \mathrm{GHz}$ due to less usage of the bandwidth. The increase in throughput comes mainly from the increase in bandwidth to deliver high data rates of video applications to user terminals. The technology supports multi-user, multi-input, multi-output (MU-MIMO) scenarios and utilizes the 256 quadrature amplitude modulation (QAM). The standard group approved TGac Channel Model Addendum v12 [2,3], which are mainly modifications of the IEEE802.11n channel models [4] for wide bandwidth. The adopted models assume fixed tapped delay line channel models with a tap spacing of $5 \mathrm{~ns}$ and with some defined directional clusters. The directional clusters parameters are defined for angle of arrival and its angular spread, and angle of departure and its angular spread for every tap within its directional cluster. 
It is important to study the impact of re-configurability of antenna pattern vertical and horizontal polarizations due to its rotation angle at client's side and antenna heights at access point side in different indoor environments that have different channel characteristics in terms of dispersion in delay and direction domains. The adopted channel model by IEEE 802.11ac, i.e., TGac does not provide the capability for such study since it has fixed delayed components that do not vary with mobile station variability. So, this work adopts physics-based model that derives each ray's parameters from environment geometry.

In this work, we present the impact of re-configurability antenna patterns due to its rotation at client side and height of access point in three different common indoor environments (lecture hall, corridor and banquet hall) on amount of fading.

\section{Radio Channel Model}

Radio wave propagation in an indoor environment is confined by the scatterers from different directions such as side walls, opposite walls, floor and ceiling. For indoor line of sight propagation, signals arrive at the receiver via direct path, signals bounce between opposite walls, side walls, ceiling and floor or any combinations between these different surfaces. This makes the signal arrive from almost all possible azimuthal angles with large directional dispersion in the vertical directional plane. These different rays would experience different antenna gains and losses as a function of angular information, azimuthal and co-elevation departure and arrival directions. The adopted channel model in this work is based on the physics of the specular reflection propagation mechanism in addition to the line of sight component. These propagation mechanisms are presented in terms of multi-rays that exhibit multi-dimensional parameters. This work models also the RF propagation in commonly shaped cubical indoor environment. This particular shape of indoor environment includes different propagation scenarios such as a corridor, office, lecture hall, convention center, etc. The tested model essentially presents similar features to the model presented in [5]. The simulated propagation characteristics are determined by the input parameters to the model and communications link setup. The input parameters to the channel model include operating frequency, system bandwidth, antenna's polarization and the heights of transmitter and receiver, antennas' field pattern, electrical properties of scatterers, etc. The communication link setup includes the locations of transmitter and receiver antennas with respect to the scatterers and reflecting surfaces such as ceiling, side and opposite walls, etc. In this physical model, each ray is determined by its parameters defined by its delay, azimuth-coelevation angle of arrival, and azimuth-co-elevation angle of departure. The complex amplitude of each ray is computed with electromagnetic formulations for free space loss and loss due to the interaction with the scatterers in the environment. The interaction loss depends on the interaction, wave-front and geometrical properties of impinging rays and physical properties of reflecting surfaces. Different coefficients can be used to capture the interaction losses that depend on the transmit waveform type: plane wave, cylindrical wave or spherical wave. The most commonly used reflection coefficient is the Fresnel plane wave reflection coefficient, which is valid for flat surfaces and is function of the incidence angle and electrical properties of the reflecting surface. The RF propagation characteristics depend on how the multi-ray components interact with each other based on their phases and amplitudes constructively or destructively to create different fading profiles. The multi-domain RF characteristics depend on the dispersion pattern in their corresponding domain such as delay, direction and Doppler.

The received signal is obtained as a sum of multi-ray components as linear superposition of $N$ individual rays, and it can be represented as follows: 
International Journal of Wireless \& Mobile Networks (IJWMN) Vol. 6, No. 2, April 2014

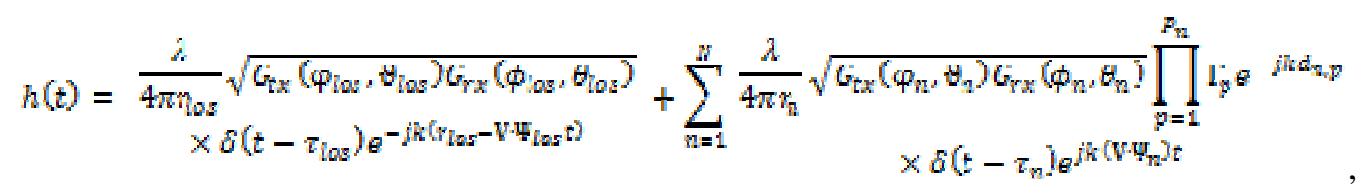

where $k$ is the wave number expressed as $k=\frac{3 \pi}{\pi}, \lambda$ is the wavelength of operating frequency, $\Gamma_{p}$ denotes the Fresnel reflection coefficient for the $p$-th wave-interface intersection, $\epsilon_{t x}\left(\varphi_{n}, \theta_{n 2}\right)$, $G_{r z}\left(\phi_{n}, \theta_{n}\right)$ are the transmitter and receiver antenna gain, respectively, $\mathbf{v}$ is the velocity (speed and direction) of the mobile terminal, which is assumed as the receiver in this notation, and defined by $V=v_{x} \vec{x}+v_{y} \vec{y}+v_{z} \vec{z}$ and $\Psi_{n}$ stands for the arrival direction vector defined for ray $n$ as $\Psi_{n}=\cos \left(\phi_{n}\right) \sin \left(\theta_{n}\right) \vec{x}+\sin \left(\phi_{n}\right) \sin \left(\theta_{n}\right) \vec{y}+\cos \left(\theta_{n}\right) \vec{z}$

where $\phi_{\mathrm{n}}$ and $\theta_{\mathrm{n}}$ are the horizontal and co-elevation arrival angles of ray $n$ (or LOS ray when subscript is los) relative to the $x$-axis and $z$-axis, respectively, and $\gamma_{\mathrm{n}}$ is the path length of ray $n$, $d_{n g}$ denotes the distance traversed by the specular wave between the $(p-1)$ and $p$-th boundary intersections, $\eta_{\text {los }}$ is the length of LOS path and $n_{\text {in }}$ is the specular reflection path length.

\section{Reconfigurable Antenna (RA)}

It can be used in a way to change radio channel characteristics in favor of enhancing performance of wireless communications system. The RA, per control, can change its field pattern, pointing direction, operating frequency and polarization. The key part in gaining benefit from RA is based on understanding the interplay between superposition of complex signals of multipath components of radio channel, three dimensional antenna patterns, and velocity of mobile terminal. This interplay would affect channel characteristics in delay, direction and Doppler domains, which will have impact on their corresponding correlation parameters such as coherence spectra (i.e., frequency correlation), spatial correlation and coherence time, respectively. The RF agility of RA can be used to change some of these channel correlation properties such as the coherence time and power weighted multipath dispersion metrics. In this work, reconfiguring antenna pattern of a mobile device is simulated in terms of rotation angle of the antenna, which changes antenna pattern for vertical polarization that can be written as [6]

$$
G_{v}(\theta, \phi)=1.64(\cos \theta \cos \phi \sin \alpha-\sin \theta \cos \alpha)^{2} \frac{\cos ^{2}(\pi \zeta / 2)}{\left(1-\zeta^{2}\right)^{2}}
$$

and its antenna gain pattern for horizontal polarization is

$$
\sigma_{\mathrm{R}}(\theta, \phi)=1.64 \sin ^{2} \phi \sin ^{2} \alpha \frac{\cos (\pi \zeta / 2)^{2}}{\left(1-\zeta^{2}\right)^{2}}
$$

where $\zeta=\sin \theta \cos \phi \sin \alpha+\cos \phi \cos \alpha$ and the angle $\alpha$ is the rotation angle of the antenna element from $z$-axis in the vertical $z x$-plane, $\phi$ is the azimuth angle relative to $x$-axis, $\theta_{\text {is }}$ the elevation angle relative to z-axis, the coefficient 1.64 corresponds to the directivity of the halfwavelength dipole antenna.

\section{Amount of Fading}

The selected measure of severity of fading in this work is the amount of fading (AF), which can be computed using the first and second central moments of SNRs at diversity output. The AF is defined in [7-9] as 
International Journal of Wireless \& Mobile Networks (IJWMN) Vol. 6, No. 2, April 2014

$$
A F=\frac{v a r\left\{\alpha^{2}\right]}{E\left[\alpha^{2}\right\}^{2}}
$$

where $\alpha$ is the instantaneous fading amplitude of a complex fading channel, $\boldsymbol{E} \boldsymbol{f} \boldsymbol{\mathcal { H }}$ and $\operatorname{var}\{\boldsymbol{\mathcal { N }}$ are the statistical mean and variance, respectively. To quantify the probability distribution of fading, it is mentioned in $[7,8]$ that for Nakagami- $m$ fading distribution of $\alpha$, the amount of fading (AF), $A F=1 / m$, whose range is $[0,2]$. When $m=\infty, A F=0$, which corresponds to the situation of "no fading". When $m=1$, the $\mathrm{AF}=1$, which corresponds to Rayleigh fading, and when $m=0.5$. $\mathrm{AF}=2$, which corresponds to the one-sided Gaussian distribution and the severest fading assumed by the Nakagami- $m$ fading channel. Hence, when $\mathrm{AF}<1$, the fading severity of the radio channel is considered less than that of Rayleigh channel and the otherwise AF $>1$. It is expected that the communication system performance degradation increases with AF. It is worth to mention that the fading parameter in line of sight (LOS) propagation, $K$ factor, of Rician fading distribution is a function of the AF. The $K$ factor is defined as the ratio of power received via LOS propagation to the power of non-LOS paths. This relationship between AF and $K$ parameter is presented in [10] as follows

$$
K=\frac{\sqrt{1-A F}}{1-\sqrt{1-A F}}
$$

Different forms of this relationships are also given in [11-13]. So, the estimation of AF can be used to indicate $K$ parameter of Rician fading channel as well as $m$ parameter of Nakagami- $m$ fading channel since $\mathrm{AF}=1 / \mathrm{m}$. The $\mathrm{AF}$ formulation in [14] is presented in closed form expression for identically distributed spatially correlated Nakagami- $m$ fading fading channel at output of at the output of a space-time block-coded multiple-input-multiple-output (MIMO) diversity system. A closed-form expression for high order amount of fading for Nakgami- $m$ fading channel is presented in [15], which was defined originally in [16].

\section{Numerical Results}

The presented results are obtained for three different indoor environments, where the IEEE802.11ac system may operate. These indoor environments are 1) lecture hall with dimensions, height $(\mathrm{H})=4 \mathrm{~m}$, width $(\mathrm{W})=8 \mathrm{~m}$, length $(\mathrm{L})=10 \mathrm{~m}, 2$ ) corridor with dimensions: $\mathrm{H}$ $=4 \mathrm{~m}, \mathrm{~W}=2 \mathrm{~m}$, and $\mathrm{L}=30 \mathrm{~m}$, and 3 ) banquet hall with dimensions: $\mathrm{H}=10 \mathrm{~m}, \mathrm{~W}=15 \mathrm{~m}$, and $\mathrm{L}=$ 50. The simulations are set-up for a Wi-Fi antenna access point (AP) at different eights $(1 / 4 \mathrm{H}, 1 / 2$ $\mathrm{H}, 3 / 4 \mathrm{H}$, and $\mathrm{H}$ ) and client station antenna height is $1.7 \mathrm{~m}$. It is assumed that $\mathrm{AP}$ is the transmitter and client station is the receiver. The receiver speed is $3 \mathrm{~km} / \mathrm{hr}$, which is defined as the pedestrian speed in 3GPP standard [17]. Number of source images per reflecting surface is set to 6 that cause multiple reflection rays in addition to the line of sight component. Reflecting surfaces have relative permittivity of 5 and conductivity of 0.02 . The simulated temporal range is for one second for every spatial location. The temporal sampling rate is 26,000 samples/sec. In order to investigate the effect of the environment on similar communication link setups, the three indoor environments have been tested for same route from the access point. A route starting from a horizontal distance of $2 \mathrm{~m}$ from AP till $9.5 \mathrm{~m}$ with spatial resolution of about $2.5 \mathrm{~cm}$ is used in the study. It is assumed that transmitter antenna is vertically polarized. Re-configurability of antenna pattern is done via controlling rotation angle. Three different rotation angles have been selected to generate three different antenna patterns for vertically polarized antennas. Figure 1 shows the three antenna patterns for vertical polarization state for three antenna states correspond to three rotation angles; $0^{\circ},-15^{\circ}$, and $-55^{\circ}$. Figure 2 shows samples of fading profile for $0^{\circ}$ rotation angle in a lecture hall for four different heights of access points. The severity of fading levels in fading profiles are computed with AF. The AF values are shown on Figure 2 as follows: 1) for AP height at $1 / 4 \mathrm{H}$, the $\mathrm{AF}=0.34,2$ ) for $\mathrm{AP}$ height $1 / 2 \mathrm{H}, \mathrm{AF}=0.22,3$ ) for $\mathrm{AP}$ height $3 / 4 \mathrm{H}$, the $\mathrm{AF}=0.6$, and 
4) for AP height $\mathrm{H}$, the $\mathrm{AF}=0.89$. Hence, it can be observed that for this particular sample, the fading profile of AP placed at ceiling has highest fading level and lowest fading level is observed when the AP is placed at height half the ceiling height. From this analysis, we see that we can map fading profiles as shown in Figure 2 from time series presentation to indices that indicate the fading severity levels. In order to get the feeling of AF numbers with severity of different fading channel models, Figure 3 shows cumulative distribution function (CDF) of envelopes of Rayleigh, double Rayleigh and Rician fading channels. The AF of Rayleigh fading is one while that of the double Rayleigh fading channel is three. Rician fading channels for every $\mathrm{k}$ parameter, it becomes Rayleigh as can be seen in Figure 3 for $\mathrm{K}=-10 \mathrm{~dB}$, while for AF decreases with increasing of $\mathrm{K}$ factor.

In order to get statistically significant results, we need to compute the AF for large number of positions within the indoor environment for different heights of AP and different rotation angles. The AF values are presented in terms of CDF. Figure 4 shows CDF of AF for antenna rotation angles in the three different indoor environments. Table I summarizes the $90^{\text {th }}$ percentile of CDF curve for rotation angle of $0^{\circ}$. It can be seen that the curve of least AF values correspond to AP of height $1 / 2 \mathrm{H}, 1 / 2$ the ceiling height, for lecture hall and corridor environments but not for banquet hall. The height of AP that correspond to lowest AF in banquet hall is the $1 / 4 \mathrm{H}$. However, the difference in the $90 \%$ percentile values of $1 / 4 \mathrm{H}$ and $1 / 2 \mathrm{H}$ for banquet hall might be considered small. Very similar observed results can be seen for clients' antenna rotation angles at $-15^{\circ}$ as depicted on Figure 5 and presented in Table II. For client antenna's rotation angle at $-55^{\circ}$, the lowest AF curve is observed to correspond to AP height on ceiling for lecture hall and corridor indoor environments, while for banquet hall, it is still the AP height of $1 / 4 \mathrm{H}$ has the lowest AF curve. This clearly shown on Figure 6. Table III presents the $90^{\text {th }}$ percentile of AF in the three indoor environments for antenna's rotation angle of $-55^{\circ}$. Lecture hall and corridor shows quite similar results of $\mathrm{AF}$ at this percentile and the value is close to that of Rayleigh fading channel. The significant corresponding values are clearly observed for banquet hall for the three AP antenna heights. It should be remembered that the curve of lowest AF corresponds to channels that have highest performance of wireless communication system. The other parameter that describes the stationarity of the fading process is the coherence time of the radio channel and how it is related to AF since AF is computed for particular time interval of channel series. Coherence time is a measure of similar behavior of radio channel over a particular period. We compute it here from autocorrelation of time series of channel envelopes at what time the normalized autocorrelation coefficient starts to be below 0.5. We extracted the coherence times for all channel positions, where we already computed the AF values. Then, we calculated the crosscorrelation level between the AF and coherence time. Table IV presents correlation levels for the three indoor environments and the four AP antenna heights for the case, when antenna's rotation angle is $0^{\circ}$. It can be seen that the highest absolute correlation levels correspond to the case when the AP antenna height is $1 / 2$ the ceiling height. The values indicate that the as the AF gets lower the coherence time gets longer, which is expected since the low AF means the that radio channel is less sever fading and stability condition is what makes the coherence time longer. The absolute correlation values for banquet hall are largest for AP heights of $1 / 4 \mathrm{H}$ and at ceiling. Their correlation values are quite similar but the curve of AF of AP height of $1 / 4 \mathrm{H}$ is smallest. Table $\mathrm{V}$ presents correlation levels between channel coherence time and AF for the three indoor environments when client antenna's rotation angle is $-15^{\circ}$. It shows very similar results as in the case of rotation angle of $0^{\circ}$. Table VI presents the corresponding correlation values for rotation angle of $-55^{\circ}$. The larges absolute correlation values is observed for the case of AP antenna is placed on ceiling of lecture hall and corridor. While for banquet hall, the largest absolute correlation value is for the case of $\mathrm{AP}$ antenna height is at $1 / 4 \mathrm{H}$. 
International Journal of Wireless \& Mobile Networks (IJWMN) Vol. 6, No. 2, April 2014

\section{CONCLUSIONS}

This work showed that impact of client antenna re-configurability in terms of its rotation angle and impact of antenna height of AP on level of AF in three different indoor environments of different dispersion characteristics. The results show that for placing antenna of AP at height $1 / 2$ of the ceiling height in lecture hall and corridor leads to least AF, which may lead to higher performance of wireless communication system. This is true when rotation angle is close to vertical polarization, i.e., $0^{\circ}$ and $-15^{\circ}$, but the effect of AP height on AF is quite small when rotation angle is $-55^{\circ}$. For banquet hall, the results show that placing AP antenna height at $1 / 4 \mathrm{H}$ shows lowest ranges of AF at antenna's rotation angles of $0^{\circ}$ and $-15^{\circ}$ and when AP is on the ceiling results in smallest values of $\mathrm{AF}$ for rotation angle of $-55^{\circ}$.

\section{ACKNOWLEDGEMENTS}

This publication was made possible by NPRP grants \#: NPRP 5-653-2-268 from the Qatar National Research Fund (a member of Qatar Foundation). The statements made herein are solely the responsibility of the authors.

\section{REFERENCES}

[1] Official IEEE 802.11 Working Group Project Timelines-2013-05-18, Online: http://grouper.ieee.org/groups/802/11/Reports/802.11_Timelines.htm.

[2] Greg Breit, et al, "TGac Channel Model Addendum", Institute of Electronic and Electrical Engineers, IEEE802.11-09/0308r12, March 18, 2010. Online: Available: https://mentor.ieee.org/802.11/dcn/09/11-09-0308-12-00ac-tgacchannel-model-addendumdocument.doc.

[3] Breit, G. et al., "TGac Channel Model Addendum Supporting Material," Doc. IEEE802.1109/0569r0. Erceg,

[4] V. et al. "TGn Channel Models." Doc. IEEE802.11-03

[5] W. Q. Malik, C. J. Stevens, and D. J. Edwards, "Spatio-temporal ultrawideband indoor propagation modelling by reduced complexity geometric optics," IET Commun., vol. 1, no. 4, pp. 751-759, 2007.

[6] T. Taga "Analysis for Mean Effective Gain of Mobile Antennas in Land Mobile Radio Environments," IEEE Trans. Veh. Technol. vol. 39, no. 2, pp. 117 - 131, 1990.

[7] U. Charash, "Reception through Nakagami multipath channels with random delays," IEEE Trans. on Commun., vol. 27, no. 4, pp. 657-670, Apr. 1979.

[8] M. Simon and M. Alouini, Digital Communications over Fading Channels: A Unified Approach to Performance, Analysis, John Wiley \& Sons, Inc. 2000.

[9] H. El-Sallabi, K. Qaraqe and Erchin Serpedin, "Some Insights on the Amount of Fading in Radio Channels" in Proc. PIERS 2013, Stockholm, Sweden, August, 2013.

[10] A. Abdi, A., C. Tepedelenlioglu, M. Kaveh, and G. Giannakis, "On the estimation of the K parameter for the Rice fading distribution”, IEEE Communications Letters, Volume 5, Number 3, March 2001, Pages 92-94.

[11] P. K. Rastogi and O. Holt, "On detecting reflections in presence of scattering from amplitude statistics with application to D region partial reflections," Radio Sci., vol. 16, pp. 1431-1443, 1981.

[12] L. J. Greenstein, D. G. Michelson, and V. Erceg, "Moment-method estimation of the Ricean Kfactor," IEEE Commun. Lett., vol. 3, pp. 175-176, 1999.

[13] P. D. Shaft, "On the relationship between scintillation index and Rician fading," IEEE Trans. Commun., vol. 22, pp. 731-732, 1974.

[14] B. Holter and G. E. Øien "On the Amount of Fading in MIMO Diversity Systems," IEEE Trans. On Wireless Commun., vol. 4, no. 5, Sep. 2005, pp. 2498 - 2507. 
International Journal of Wireless \& Mobile Networks (IJWMN) Vol. 6, No. 2, April 2014

[15] A. Hyadi, M. Benjillali, M.-S. Alouini, and D. B. da Costa, "Performance Analysis of Underlay Cognitive Multihop Regenerative Relaying Systems with Multiple Primary Receivers" IEEE Trans. Wireless Commun., vol. 12, pp. 6418-6429, 2012.

[16] F. Yilmaz and M.-S. Alouini, "Novel asymptotic results on the high order statistics of the channel capacity over generalized fading channels," in Proc. 2012 International Workshop on Signal Processing Advances in Wirless Communications, pp. 389-393.

Table I. The $90^{\text {th }}$ percentile of AF for antenna's rotation angle of $0^{\circ}$.

\begin{tabular}{|c|c|c|c|c|}
\hline & $1 / 4 \mathrm{H}$ & $1 / 2 \mathrm{H}$ & $3 / 4 \mathrm{H}$ & $\mathrm{H}$ \\
\hline Lecture Hall & 1.14 & 0.81 & 1.21 & 1.14 \\
\hline Corridor & 0.89 & 0.75 & 0.96 & 1.10 \\
\hline Banquet Hall & 0.71 & 0.84 & 1.03 & 1.11 \\
\hline
\end{tabular}

Table II. The $90^{\text {th }}$ percentile of AF for antenna's rotation angle of $-15^{\circ}$.

\begin{tabular}{|c|c|c|c|c|}
\hline & $1 / 4 \mathrm{H}$ & $1 / 2 \mathrm{H}$ & $3 / 4 \mathrm{H}$ & $\mathrm{H}$ \\
\hline Lecture Hall & 1.17 & 0.84 & 1.14 & 1.02 \\
\hline Corridor & 0.92 & 0.79 & 0.89 & 0.98 \\
\hline Banquet Hall & 0.66 & 0.69 & 0.79 & 0.83 \\
\hline
\end{tabular}

Table III. The $90^{\text {th }}$ percentile of AF for antenna's rotation angle of $-55^{\circ}$.

\begin{tabular}{|c|c|c|c|c|}
\hline & $1 / 4 \mathrm{H}$ & $1 / 2 \mathrm{H}$ & $3 / 4 \mathrm{H}$ & $\mathrm{H}$ \\
\hline Lecture Hall & 1.03 & 1.03 & 1.06 & 0.99 \\
\hline Corridor & 0.95 & 1.10 & 0.93 & 0.99 \\
\hline Banquet Hall & 0.74 & 0.56 & 0.44 & 0.42 \\
\hline
\end{tabular}

Table IV. Correlation value between channel coherence time and AF for antenna's rotation angles of $0^{\circ}$.

\begin{tabular}{|c|c|c|c|c|}
\hline & $1 / 4 \mathrm{H}$ & $1 / 2 \mathrm{H}$ & $3 / 4 \mathrm{H}$ & $\mathrm{H}$ \\
\hline Lecture Hall & -0.55 & -0.62 & -0.5 & -0.33 \\
\hline Corridor & -0.68 & -0.79 & -0.69 & -0.65 \\
\hline Banquet Hall & -0.57 & -0.34 & -0.50 & -0.60 \\
\hline
\end{tabular}

Table V. Correlation value between channel coherence time and AF for antenna's rotation angles of $-15^{\circ}$.

\begin{tabular}{|c|c|c|c|c|}
\hline & $1 / 4 \mathrm{H}$ & $1 / 2 \mathrm{H}$ & $3 / 4 \mathrm{H}$ & $\mathrm{H}$ \\
\hline Lecture Hall & -0.57 & -0.61 & -0.46 & -0.42 \\
\hline Corridor & -0.64 & -0.78 & -0.69 & -0.70 \\
\hline Banquet Hall & -0.61 & -0.43 & -0.51 & -0.61 \\
\hline
\end{tabular}

Table VI. Correlation value between channel coherence time and AF for antenna's rotation angles of $-55^{\circ}$.

\begin{tabular}{|c|c|c|c|c|}
\hline & $1 / 4 \mathrm{H}$ & $1 / 2 \mathrm{H}$ & $3 / 4 \mathrm{H}$ & $\mathrm{H}$ \\
\hline Lecture Hall & -0.48 & -0.54 & -0.60 & -0.81 \\
\hline Corridor & -0.40 & -0.75 & -0.46 & -0.73 \\
\hline Banquet Hall & -0.69 & -0.65 & -0.63 & -0.20 \\
\hline
\end{tabular}


International Journal of Wireless \& Mobile Networks (IJWMN) Vol. 6, No. 2, April 2014

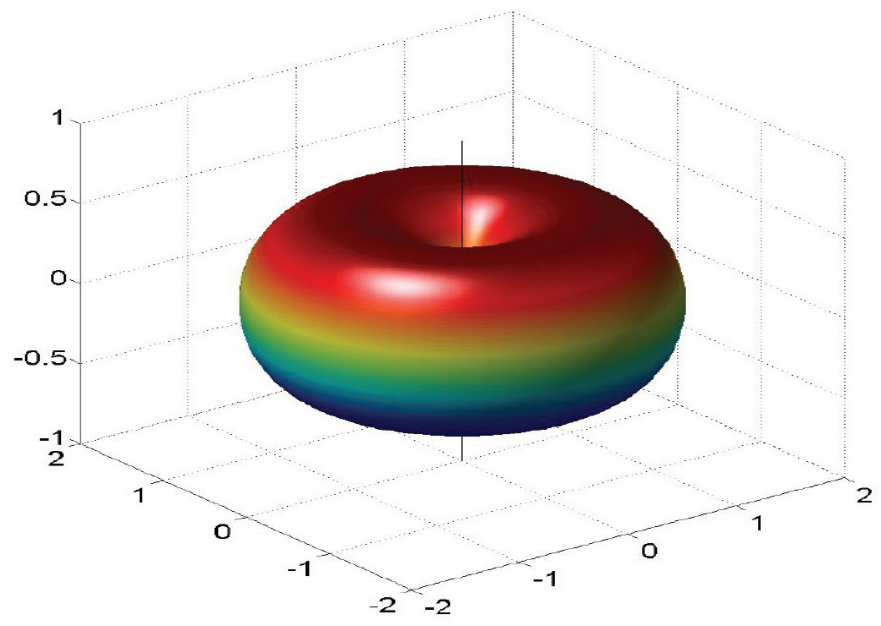

a. $\quad$ Rotation angle $=0^{\circ}$

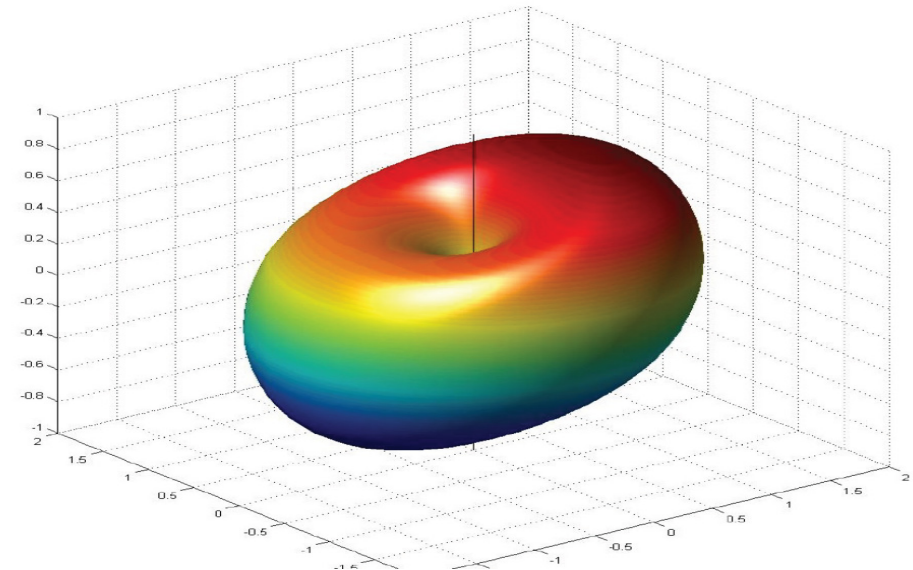

b. Rotation angle $=-15^{\circ}$

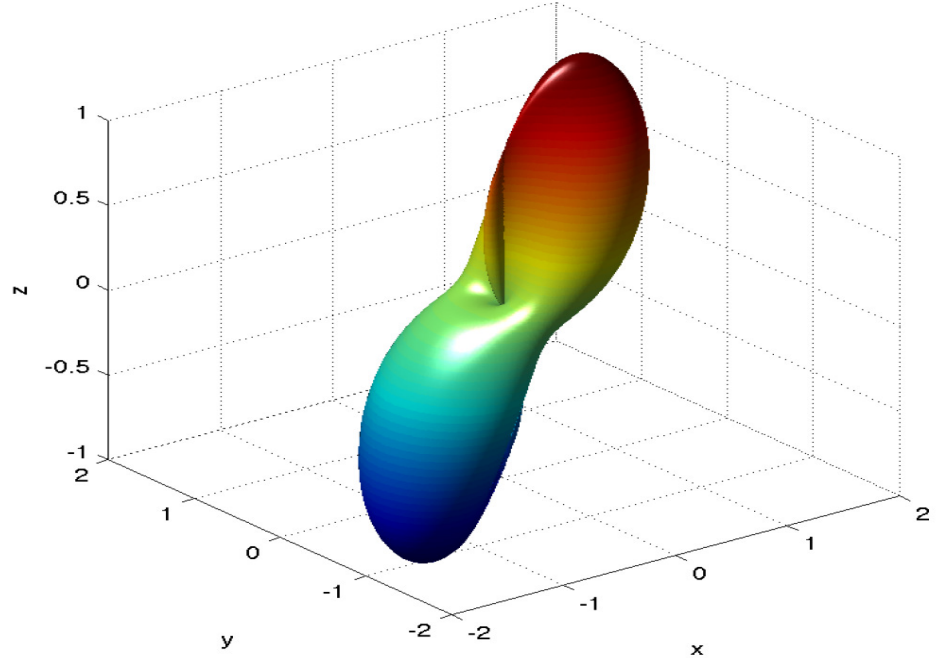

a. Rotation angle $=-55^{\circ}$

Figure 1. Antenna pattern for vertical polarization at different antenna's rotation angles 
International Journal of Wireless \& Mobile Networks (IJWMN) Vol. 6, No. 2, April 2014

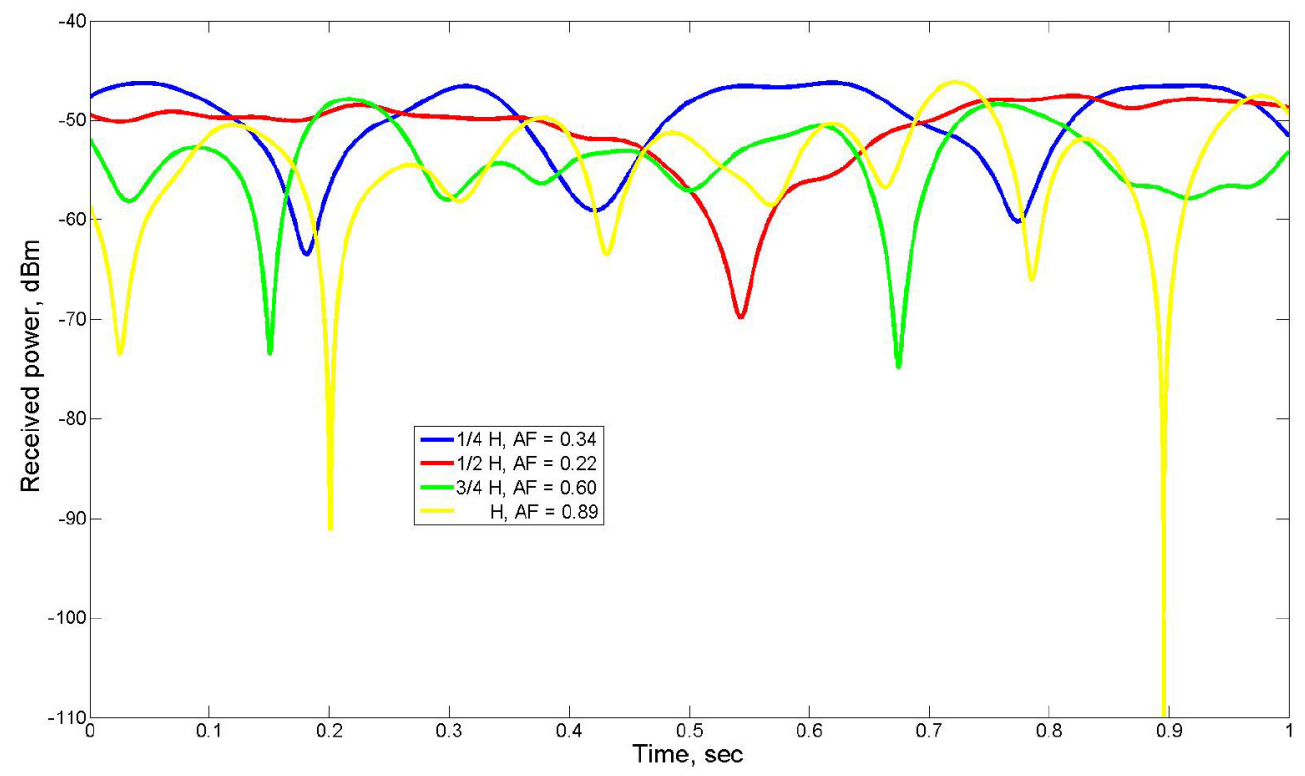

. Figure 2. Samples of fading profiles and corresponding AF values

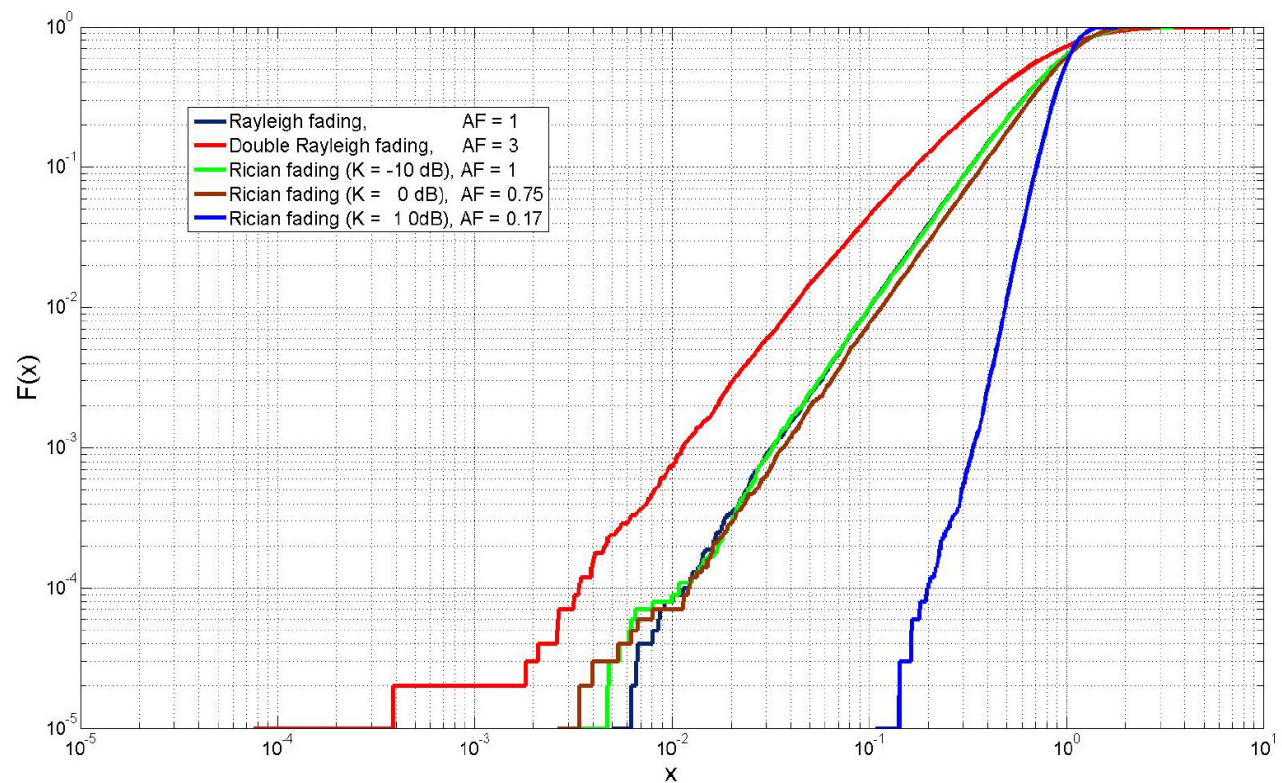

Figure 3. CDF of different common fading channels with their corresponding AF values. 
International Journal of Wireless \& Mobile Networks (IJWMN) Vol. 6, No. 2, April 2014

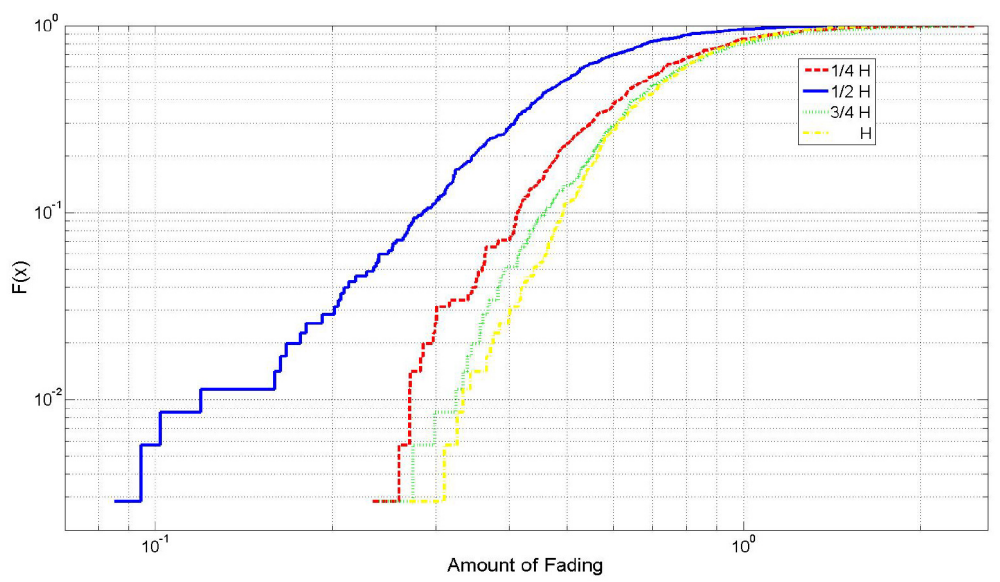

a. Lecture Hall

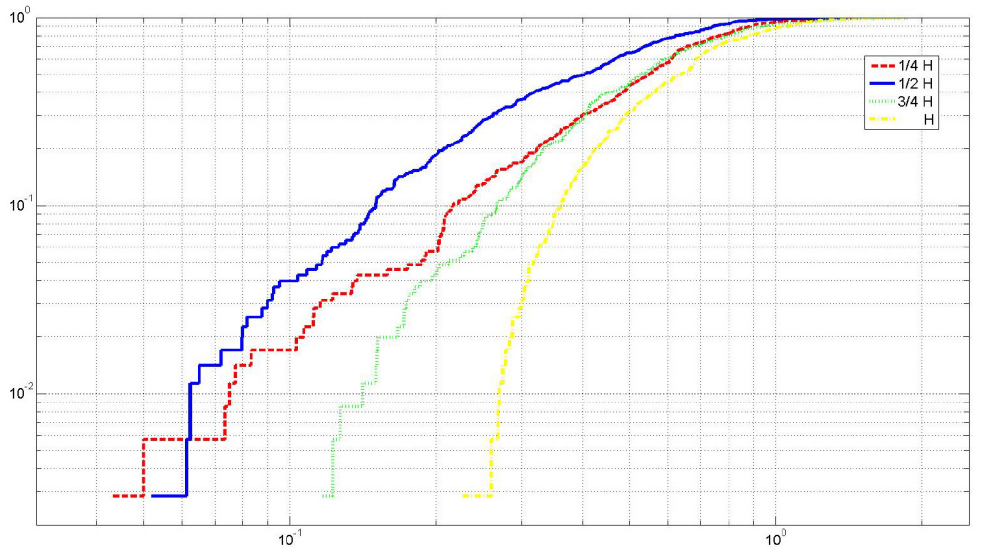

b. Corridor

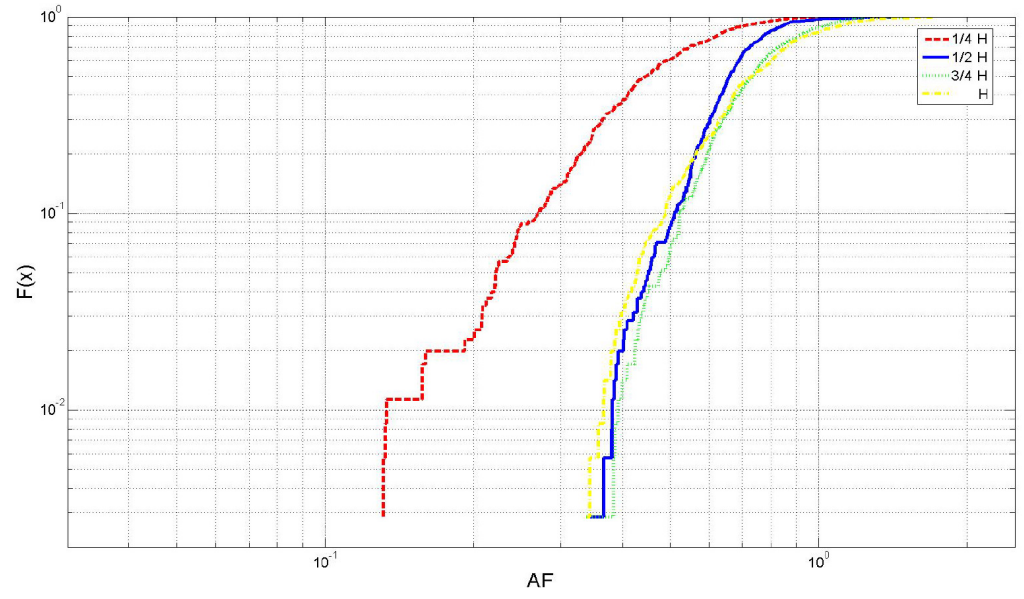

c. Banquet Hall

Figure 4. Impact of antenna height of AP on AF in three different indoor environment for 0o rotation angle of antenna at mobile station 
International Journal of Wireless \& Mobile Networks (IJWMN) Vol. 6, No. 2, April 2014

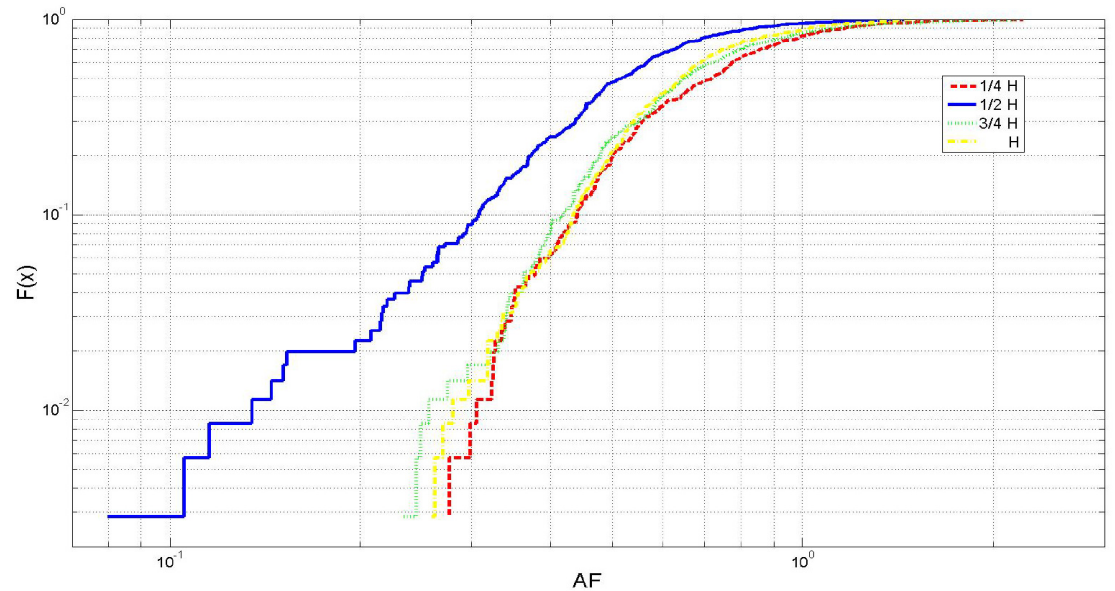

a. Lecture Hall

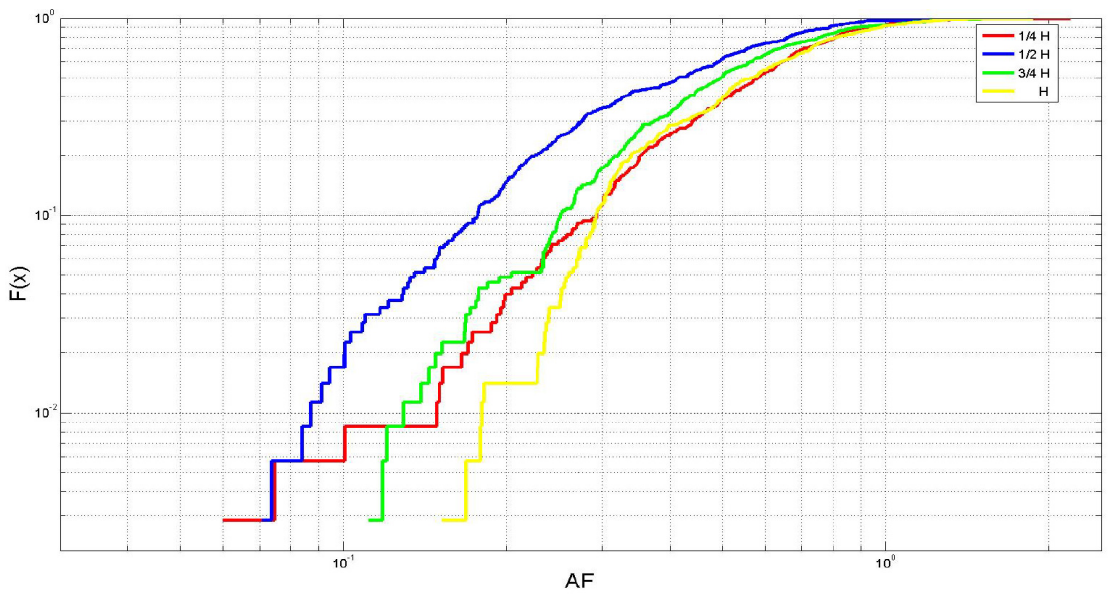

b. Corridor

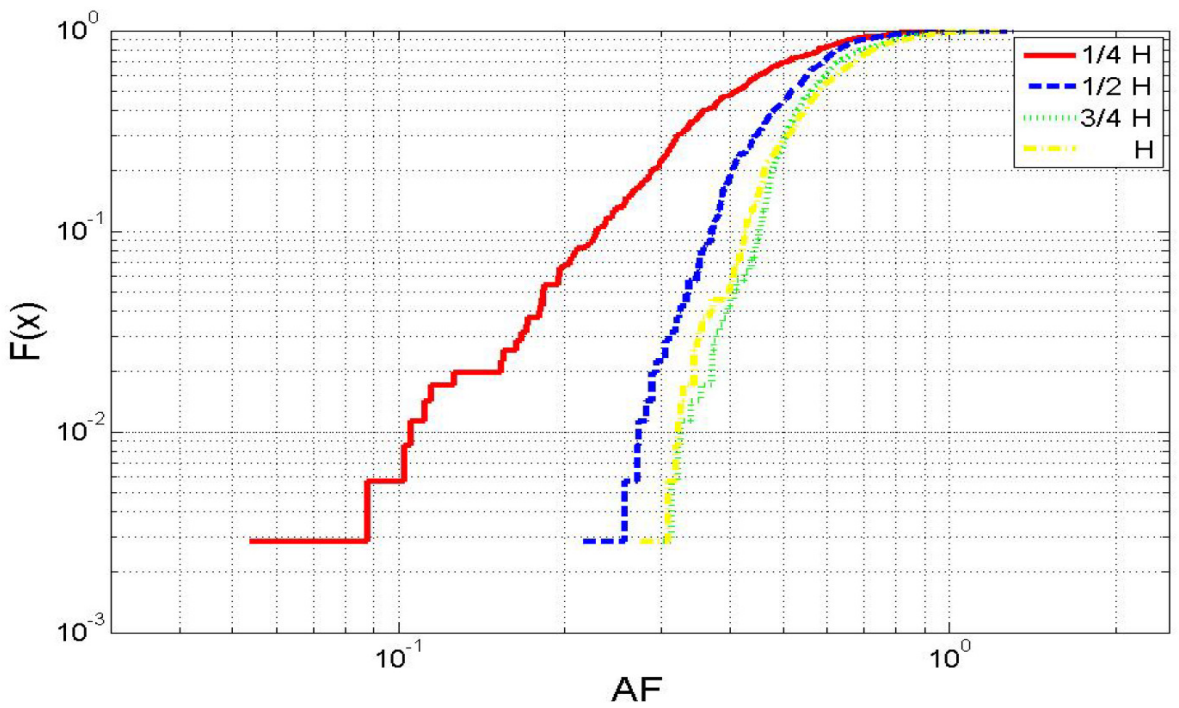

c. Banquet Hall

Figure 5. Impact of antenna height on AF in three different indoor environment for $-15^{\circ}$ rotation angle of antenna at mobile station. 
International Journal of Wireless \& Mobile Networks (IJWMN) Vol. 6, No. 2, April 2014

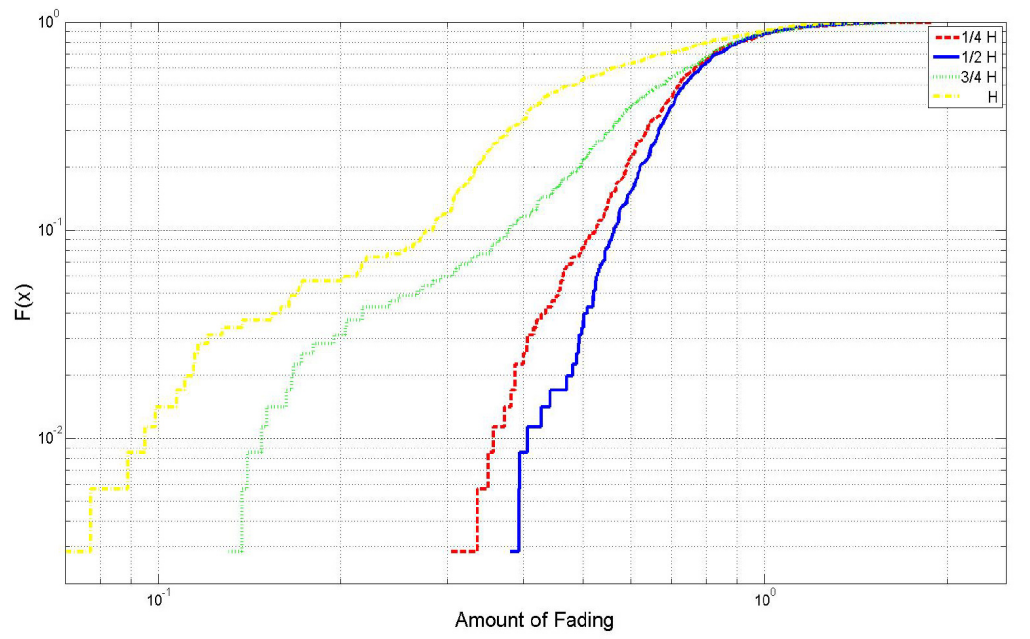

a. Lecture Hall

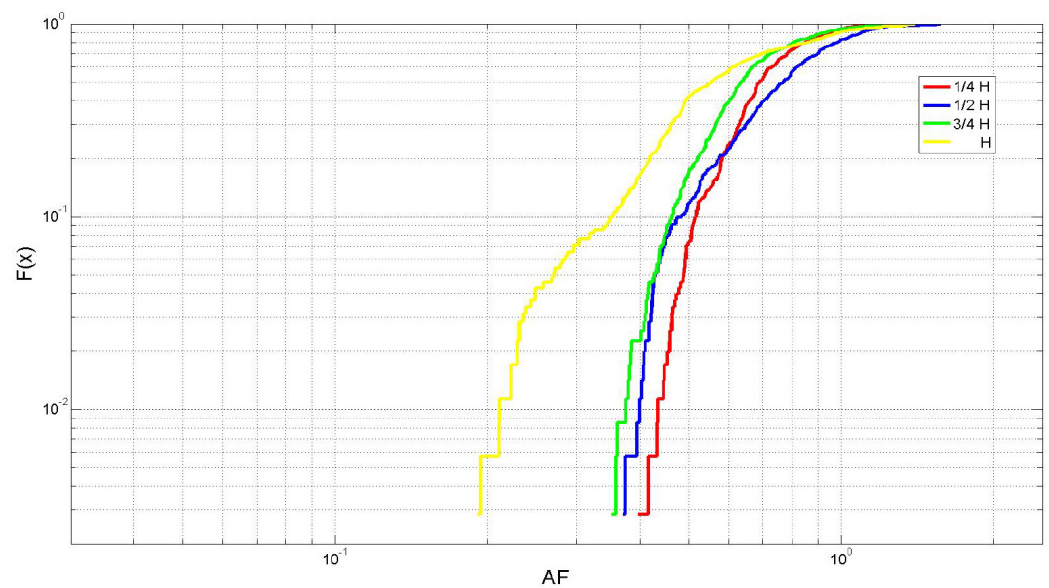

b. Corridor

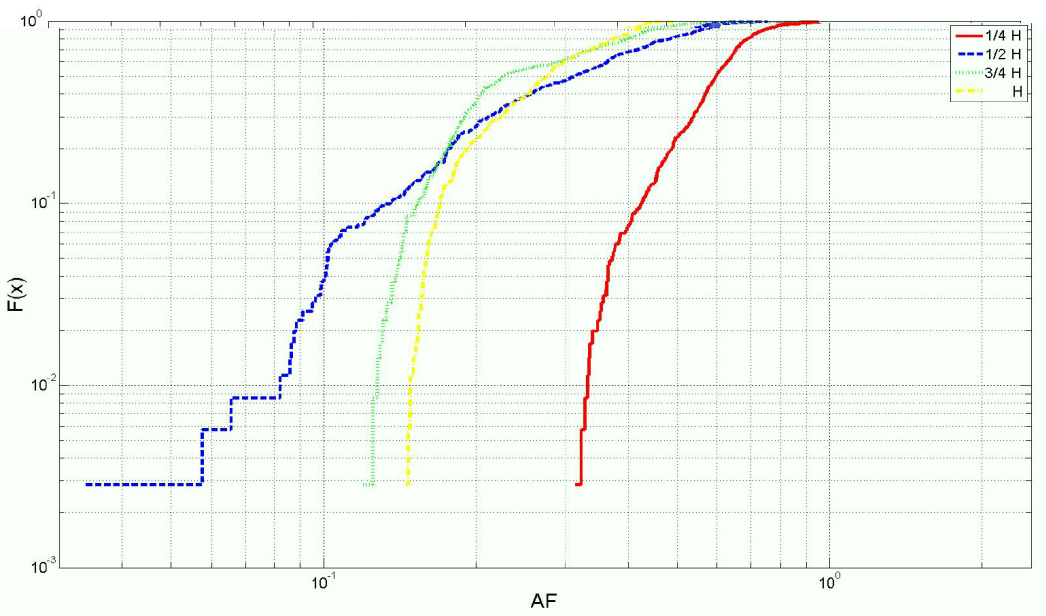

c. Banquet Hall

Figure 6. Impact of antenna height on AF in three different indoor environment for $-55^{\circ}$ rotation angle of antenna at mobile station. 
Jehad M. Hamamra was born in Nablus, Palestine, in 1990. He received the B.E. degree in electrical \& telecommunication engineering from An-Najah National University, Nablus, Palestine, in 2013. In 2012, he joined Jawwal company to make an extensive research project in the area of LTE Physical Layer Simulation Focusing on indoor/outdoor propagation models (WINNER channel), frequency reuse techniques and Self-Organizing Schedulers for different services Using Neural Network". In 2013, he joined the

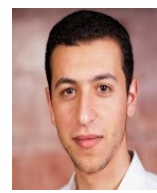
Department of Electrical \& Computer Engineering, Texas A\&M University at Qatar (TAMUQ), as a trainee researcher. His current research interests include reconfigurable antenna at mobile wireless communication system, channel modeling and characterization, self-organizing network for LTE (4G), VLC (5G) or what is called Li-Fi technology, advance adaptive modulation for fading channels, \& cloud computing with web based applications. Hamamra has obtained many international certificates in the area of computer networks \& system simulations such as CCNA \& CST. Recently, he has established with his Telecommunication \& Computer Engineers' mates a software association called TELENG ORG, which provides web based applications \& cloud computing solutions.

Hassan El-Sallabi:

Khalid A. Qaraqe (M'97, SM'00) was born in Bethlehem. Dr Qaraqe received the B.S. degree in EE from the University of Technology, in 1986, with honors. He received the M.S. degree in EE from the University of Jordan, Jordan, in 1989, and he earned his Ph.D. degree in EE from Texas A\&M University, College Station, TX, in 1997. From 1989 to $2004 \mathrm{Dr}$ Qaraqe has held a variety positions in many companies and he has over 12 years of

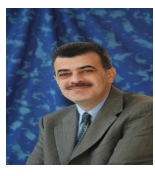
experience in the telecommunication industry. Dr Qaraqe has worked for Qualcomm, Enad Design Systems, Cadence Design Systems/Tality Corporation, STC, SBC and Ericsson. He has worked on numerous GSM, CDMA, WCDMA projects and has experience in product development, design, deployments, testing and integration. Dr Qaraqe joined the department of Electrical and Computer Engineering of Texas A\&M University at Qatar, in July 2004, where he is now a professor. Dr Qaraqe research interests include communication theory and its application to design and performance, analysis of cellular systems and indoor communication systems. Particular interests are in mobile networks, broadband wireless access, cooperative networks, cognitive radio, diversity techniques and beyond $4 \mathrm{G}$ systems. 